\title{
Is Primary Androgen Deprivation Therapy a Suitable Option for Asian Patients With Prostate Cancer Compared With Radical Prostatectomy?
}

\author{
U-Syn Ha, MD, PhDa,b; Jin Bong Choi, MDc; Jung Im Shim, MSd; Minjoo Kang, MS d; Eunjung Park, PhDd; \\ Shinhee Kang, $\mathrm{PhD}^{\mathrm{d}}$; Jooyeon Park, MSd; Jangmi Yang, MSd; Insun Choi, $\mathrm{PhD}^{\mathrm{d}}$; Jeonghoon Ahn, PhDe; \\ Cheol Kwak, MD, PhDf; Chang Wook Jeong, MD, PhDf; Choung Soo Kim, MD, PhD;; Seok-Soo Byun, MD, PhDh; \\ Seong II Seo, MD, PhD; ; Hyun Moo Lee, MD, PhDi; Seung-Ju Lee, MD, PhDi; Seung Hwan Lee, MD, PhD*; \\ Byung $\mathrm{Ha}$ Chung, MD, PhD ${ }^{\mathrm{k}}$; and Ji Youl Lee, MD, PhD
}

\begin{abstract}
Background: We conducted a comparative survival analysis between primary androgen deprivation therapy (PADT) and radical prostatectomy (RP) based on nationwide Korean population data that included all patients with prostate cancer. Materials and Methods: This study enrolled 4,538 patients with prostate cancer from the National Health Insurance Service (NHIS) database linked with Korean Central Cancer Registry data who were treated with PADT or RP between January 1, 2007, and December 31, 2014. Kaplan-Meier and multivariate survival analyses stratified by stage (localized and locally advanced) and age ( $<75$ and $\geq 75$ years) were performed using a Cox proportional hazards model to evaluate treatment effects. Results: Among 18,403 patients from the NHIS database diagnosed with prostate cancer during the study period, 4,538 satisfied inclusion criteria and were included in the analyses. Of these, 3,136 and 1,402 patients underwent $\mathrm{RP}$ or received PADT, respectively. Risk of death was significantly increased for patients who received PADT compared with those who underwent RP in the propensity score-matched cohort. In subgroup analyses stratified by stage and age, in every subgroup, patients who received PADT had a significantly increased risk of death compared with those who underwent RP. In particular, a much greater risk was observed for patients with locally advanced prostate cancer. Conclusions: Based on a nationwide survival analysis of nonmetastatic prostate cancer, this study provides valuable clinical implications that favor RP over PDAT for treatment of Asian populations. However, the possibility that survival differences have been overestimated due to not accounting for potential confounding characteristics must be considered.
\end{abstract}

J Natl Compr Canc Netw 2019;17(5):441-449 doi: 10.6004/jnccn.2018.7265

\section{Background}

Although major groups or guidelines do not recommend primary androgen deprivation therapy (PADT) as primary treatment of nonmetastatic prostate cancer, ${ }^{1,2}$ a considerable number of physicians have used PADT for nonmetastatic prostate cancer as an alternative to definitive therapies, such as radical prostatectomy (RP) and radiation therapy. ${ }^{3-5}$ This trend of using ADT as the primary treatment choice is more pronounced among elderly patients. In an analysis based on the CaPSURE registry, older patients (aged $>80$ years) were more likely than the younger population to receive PADT and had the highest rates of PADT use, followed by those aged $<60$ years (odds ratio, 5.9) and those aged 70 to 79 years (odds ratio, 2.0). ${ }^{6}$

In a study based on a Japanese population, patients with localized and locally advanced prostate cancer treated with PADT were likely to have a life expectancy similar to that of the normal population. ${ }^{7}$ Therefore, the authors suggested that, at least for older men, PADT could represent a valid therapeutic option in this patient group. ${ }^{7,8}$

Outcomes with PADT are quite different between Japanese and American populations, with one study

\footnotetext{
aDepartment of Urology, Seoul St. Mary's Hospital, College of Medicine, Seoul 'The Cancer Research Institute, The Catholic University of Korea, Seoul; 'Department of Urology, Bucheon St. Mary's Hospital, College of Medicine, The Catholic University of Korea, Bucheon; ${ }^{\mathrm{N}}$ National Evidence-based Healthcare

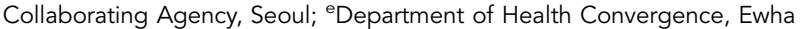
Womans University, Seoul; 'Department of Urology, Seoul National University Hospital, Seoul; ${ }^{9}$ Department of Urology, Asan Medical Center, University of Ulsan College of Medicine, Seoul; ' Department of Urology, Seoul National University Bundang Hospital, Seongnam; 'Department of Urology, Samsung Medical Center, Sungkyunkwan University School of Medicine, Seoul; jDepartment of Urology, St. Vincent's Hospital, College of Medicine, The Catholic University of Korea, Suwon; and ${ }^{\mathrm{k}}$ Department of Urology, Urological Science Institute, Yonsei University College of Medicine, Seoul, Republic of Korea.
} 
showing a considerably reduced adjusted hazard ratio for all-cause mortality of 0.27 favoring patients in Japan compared with those in the United States. ${ }^{9}$ In addition, ADT seems to be better tolerated in Japanese populations compared with Western populations in terms of bone loss, cardiovascular risk, and other factors. ${ }^{10}$

Based on these results, the NCCN Asia Consensus Statement for Prostate Cancer endorses the notion that PADT is more effective in Asian populations than in Western populations and can be considered an acceptable treatment option for most men with prostate cancer. ${ }^{11}$ Given the decreased toxicity of ADT in Asian patients, the benefits of PADT may outweigh the risks of surgery, considering the potential surgical complications, sequelae, and costs. ${ }^{12}$

However, this thesis statement for the role of PADT must be verified and strengthened with additional evidence, because most of the quoted evidence was obtained from studies conducted in a single population of Japanese patients. To confirm the role of PADT for localized and locally advanced prostate cancer, it is important to obtain clinical research results, especially results from a comparative study of PADT versus definitive treatment. Therefore, we conducted a comparative survival analysis between patients treated with PADT or RP based on nationwide Korean population data that included all patients with prostate cancer.

\section{Materials and Methods}

This study was a population-based retrospective analysis that used the National Health Insurance Service (NHIS) database to identify patients diagnosed with prostate cancer between January 1, 2007, and December 31,2009 . To analyze the survival of patients who used the medical service for prostate cancer, we used the national health insurance (NHIS-2016-1-046) database. We used insurance claim codes to define treatment modalities for patients with prostate cancer, such as prostatectomy and ADT. To accurately extract patients diagnosed with prostate cancer, this study was subsequently linked to Korean Central Cancer Registry (KCCR) data based on claims data from the NHIS. In Korea, the NHIS is the universal coverage health insurance system for the entire population and is a single-insurer system. The NHIS database represents the entire Korean population; therefore, it can be used in population-based nationwide studies of various diseases.

The KCCR has collected cancer incidence data for the entire nation by compiling a nationwide hospitalbased database. The KCCR contains official information identifying a patient's cancer stage in Korea; the summarized stage, as defined by the SEER program, was classified in the KCCR as localized (invasive cancer confined to the organ of origin), locally advanced (spread to adjacent organs and/or regional lymph nodes by direct extension), distant (extension to organs other than those covered in the regional category or metastases to distant organs or distant lymph nodes), or unknown.

Our study initially included all adults with a primary diagnosis of prostate cancer (ICD-10 code C61) identified from KCCR and NHIS data. We used NHIS codes to confirm the past history of patients with prostate cancer, and comorbidities were defined using the Charlson comorbidity index (CCI). We excluded patients who never had a cancer claim covered by insurance within a minimum of 1 year from the index date. Patients previously diagnosed with other cancers at baseline or who had previously received treatments for prostate cancer, such as chemotherapy, radiation therapy, or orchiectomy, were also excluded.

To clearly identify the treatment modality, we excluded patients who had not undergone prostate cancer biopsy, had no claim code for treatment within 6 months, and previously received other treatment for prostate cancer. Patients who received systemic chemotherapy or radiation therapy for other cancers at the index date were also excluded. In addition, to include only patients with localized or locally advanced prostate cancer, we excluded those with metastatic prostate cancer. The primary treatment groups for prostate cancer were divided into RP, which involved surgery without other treatment, and PADT, which involved hormone treatment with luteinizing hormone-releasing hormone agonists or an antiandrogen drug within the first 6 months after cancer diagnosis.

Propensity score matching (PSM) was performed to compare the PADT and RP groups. The propensity score was estimated using age, summary cancer stage, underlying disease, body mass index (BMI), and healthrelated behaviors, such as smoking, drinking, and physical activity. Because placement in the PADT group was strongly associated with patient characteristics, we used PSM analysis to balance covariates between the groups. We stratified by age and summary cancer stages to account for the clinical characteristics of prostate cancer, which were matched 1:1 with a propensity score. We also used a caliper width of 0.2 of the pooled SD of the logit of the propensity score for PSM.

We conducted stratified analyses based on the summary cancer stage and patient age to assess whether the association between PADT and mortality differed among clinical subgroups. Cancer summary stage was used to define 2 groups: those with localized prostate cancer and those with locally advanced prostate cancer. Patients were also classified into 2 groups based on age at primary treatment of prostate cancer: $<75$ and $\geq 75$ years.

Patients diagnosed with prostate cancer on December 1, 2007, were tracked through December 1, 2012, to 
account for different lengths of time in individual cohort entry dates. The primary outcome was all-cause death. The prostate cancer cohort entry date for each patient was defined as the first prostate cancer diagnosis date. Patients were followed for 5 years, and we calculated the time from the entry date to censoring or death. We defined survival time as the interval between the date of diagnosis and the date of death or end of data availability (December 31, 2014), whichever occurred first. Patients not recorded in the additional health administrative claims data after the first treatment were censored on the last administrative claims date. For survival analysis of the use of PADT and RP in patients with prostate cancer, outcome variables were defined as mortality, such as all-cause mortality, based on the cause of death reported in administration data from the National Statistical Office.

The 2-sided $t$ test was used to conduct between-group comparisons of continuous data, and the chi-square test was used for categorical data. The primary outcome of mortality rate was calculated from the date of primary treatment initiation to the date of last follow-up or death of any cause using Kaplan-Meier (KM) survival analysis and log-rank tests. A multivariate survival analysis was performed using the Cox proportional hazards model to evaluate the treatment effect with adjustment for sex, age, BMI, health behavior, and comorbidities.

Statistical significance of all analysis results was verified at a significance level of $5 \%$, and all data manipulation and statistical analyses were performed using SAS 9.4 (SAS Institute, Inc.). This study was approved by the Institutional Review Board of the National Evidence-based Healthcare Collaborating Agency (NECAIRB 16-008).

\section{Results}

Of the 4,538 patients with prostate cancer included in the prematched cohort, 3,136 underwent RP, and 1,402 received PADT (Figure 1). Median follow-up durations were 1,761 days (range, 5-1,800 days) for the study cohort, 1,758 days (range, 5-1,800 days) for patients who underwent RP, and 1,766 days (range, 5-1,799 days) for those who received PADT. In the pre-PSM cohort, 147 patients in the RP group and 389 in the PADT group died during the follow-up period (between the beginning of 2007 and the end of 2015).

\section{Patient Characteristics Before and After PSM}

Overall patient characteristics of the study cohorts pre- and post-PSM are summarized in Table 1 and supplemental eTables 1 and 2 (available with this article at JNCCN.org). In the pre-PSM cohort, significant baseline differences in age, CCI, and BMI were noted between groups. Patients in the PADT cohort were

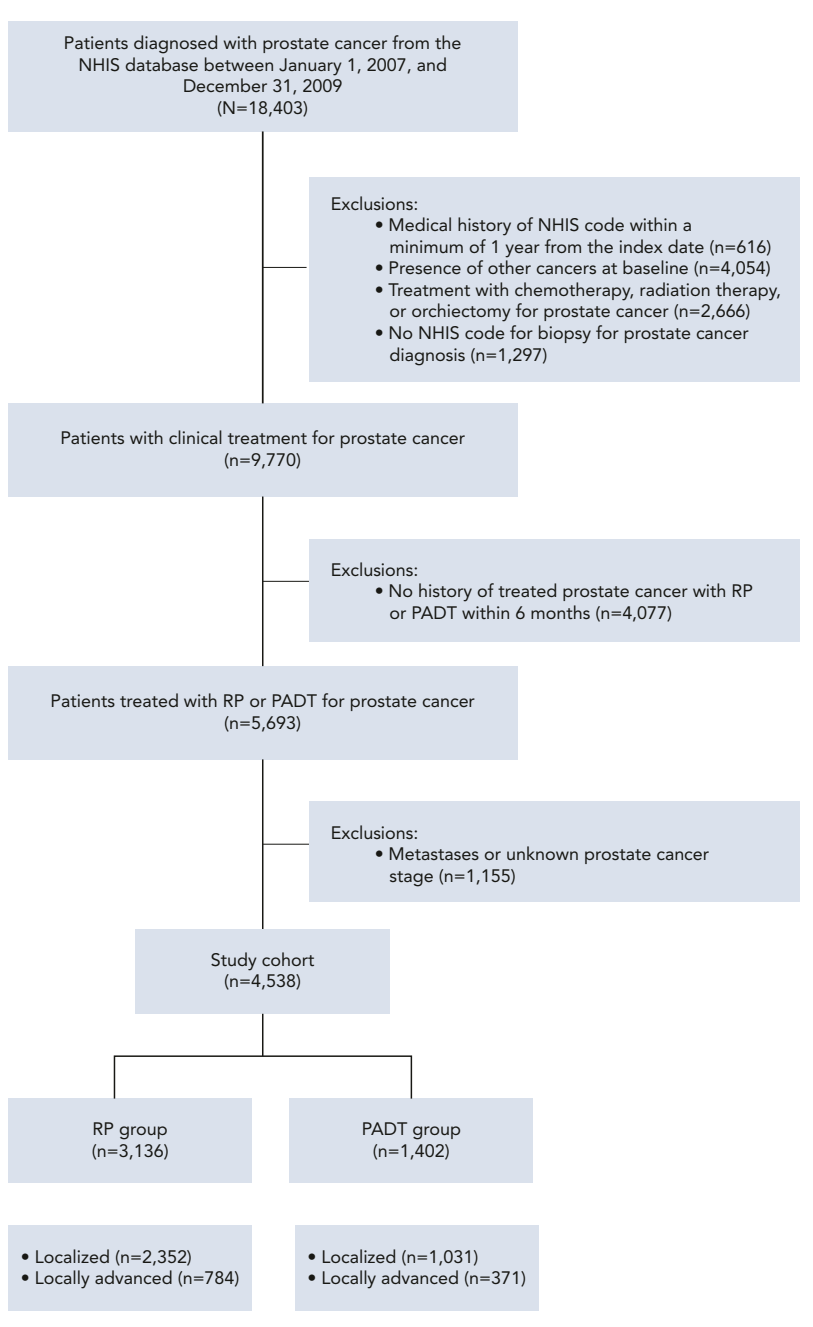

Figure 1. CONSORT diagram.

Abbreviations: NHIS, National Health Insurance Service; PADT, primary androgen deprivation therapy; RP, radical prostatectomy.

more likely to be older and have higher CCI values but were not significantly different regarding stage distribution.

After PSM, there were 956 patients in each cohort and the groups exhibited comparable baseline variable values (Table 1). Age distribution and overall health status based on CCI were also comparable between the 2 cohorts, and each had exactly the same number of patients in the cancer stage groups.

\section{Analysis of Mortality Outcomes Based on the NHIS Database}

According to KM estimates, in the pre-PSM cohort, 5 -year survival rates were $72.0 \%$ in the PADT group and $95.2 \%$ in the RP group (Figure $2 \mathrm{~A}$ ) (log-rank $P<.0001$ ). In the post-PSM matched cohort, 5-year survival rates were $77.7 \%$ in the PADT group and $92.4 \%$ in the RP group (Figure 2B) (log-rank $P<.0001$ ). 
Table 1. Distribution of Baseline Characteristics Before and After Propensity Score Matching

\begin{tabular}{|c|c|c|c|c|c|c|c|c|}
\hline \multirow[b]{2}{*}{ Variable } & \multicolumn{3}{|c|}{ Before Matching $(\mathrm{N}=4,538)$} & \multirow[b]{2}{*}{ STD } & \multicolumn{3}{|c|}{ After Matching $(\mathrm{N}=1,912)$} & \multirow[b]{2}{*}{ STD } \\
\hline & $\begin{array}{c}\operatorname{RP}(\%) \\
(n=3,136)\end{array}$ & $\begin{array}{l}\text { PADT (\%) } \\
(n=1,402)\end{array}$ & $P$ Value & & $\begin{array}{c}\mathrm{RP}(\%) \\
(n=956)\end{array}$ & $\begin{array}{c}\text { PADT (\%) } \\
(n=956)\end{array}$ & $P$ Value & \\
\hline \multicolumn{3}{|l|}{ Age group, y } & \multirow[t]{5}{*}{$<.0001$} & & & & \multirow[t]{5}{*}{.9376} & \\
\hline$<60$ & $570(18.2)$ & $71(5.1)$ & & 0.418 & $63(6.6)$ & $67(7.0)$ & & -0.017 \\
\hline 70-74 & $673(21.5)$ & $403(28.7)$ & & -0.169 & $380(39.8)$ & $382(40.0)$ & & -0.004 \\
\hline $75-79$ & $150(4.8)$ & $352(25.1)$ & & -0.595 & $140(14.6)$ & $142(14.9)$ & & -0.006 \\
\hline$\geq 80$ & $14(0.5)$ & $220(15.7)$ & & -0.583 & $13(1.4)$ & $11(1.2)$ & & 0.019 \\
\hline$<75$ & $2,972(94.8)$ & $830(59.2)$ & $<.0001$ & 0.932 & $803(84.0)$ & $803(84.0)$ & 1.0000 & 0.000 \\
\hline Locally advanced & $784(25.5)$ & $371(26.5)$ & .2961 & & $260(27.2)$ & $260(27.2)$ & 1.0000 & \\
\hline \multicolumn{3}{|l|}{$\mathrm{CCl}$} & \multirow[t]{6}{*}{.0009} & & & & \multirow[t]{6}{*}{.1068} & \\
\hline 0 & $798(25.5)$ & $328(23.4)$ & & 0.048 & $228(23.9)$ & $252(26.4)$ & & -0.058 \\
\hline 1 & $863(27.5)$ & $365(26.0)$ & & 0.034 & $242(25.3)$ & $247(25.8)$ & & -0.012 \\
\hline 2 & $676(21.6)$ & $269(19.2)$ & & 0.059 & $225(23.5)$ & $178(18.6)$ & & 0.121 \\
\hline 3 & $392(12.5)$ & $202(14.4)$ & & -0.056 & $121(12.7)$ & $123(12.9)$ & & -0.006 \\
\hline$\geq 4$ & $407(13.0)$ & $238(17.0)$ & & -0.112 & $140(14.6)$ & $156(16.3)$ & & -0.046 \\
\hline BMI, $\mathrm{kg} / \mathrm{m}^{2}($ mean $\pm \mathrm{SD})$ & $24.07(2.7)$ & $23.68(2.8)$ & .0002 & 0.146 & $23.7(2.8)$ & $23.8(2.7)$ & .2213 & -0.068 \\
\hline \multicolumn{3}{|l|}{$\mathrm{BMI}, \mathrm{kg} / \mathrm{m}^{2}$} & $<.0001$ & & & & .9055 & \\
\hline \multicolumn{3}{|l|}{ Hyperlipidemia } & \multirow[t]{3}{*}{.0006} & & & & .9603 & \\
\hline No & $2,069(66.0)$ & 997 (71.1) & & -0.111 & $665(69.6)$ & $666(69.7)$ & & -0.002 \\
\hline Yes & $1,067(34.0)$ & $405(28.9)$ & & 0.111 & $291(30.4)$ & $290(30.3)$ & & 0.002 \\
\hline Liver disease & & & .2039 & & & & .4883 & \\
\hline No & $2,322(74.0)$ & $1,063(75.8)$ & & -0.041 & $730(76.4)$ & $717(75.0)$ & & 0.032 \\
\hline Yes & $814(26.0)$ & $339(24.2)$ & & 0.041 & $226(23.6)$ & $239(25.0)$ & & -0.032 \\
\hline Diabetes & & & .1069 & & & & .7217 & \\
\hline No & $2,309(73.6)$ & $1,000(71.3)$ & & 0.052 & $691(72.3)$ & $684(71.6)$ & & 0.016 \\
\hline Yes & $827(26.4)$ & $402(28.7)$ & & -0.052 & $265(27.7)$ & $272(28.5)$ & & -0.016 \\
\hline Cardiovascular disease & & & $<.0001$ & & & & .5407 & \\
\hline No & $2,377(75.8)$ & $967(69.0)$ & & 0.153 & 695 (72.7) & $683(71.4)$ & & 0.028 \\
\hline Yes & $759(24.2)$ & 435 (31.0) & & -0.153 & $261(27.3)$ & $273(28.6)$ & & -0.028 \\
\hline
\end{tabular}

(continued on next page) Abbreviations: BMI, body mass index; CCl, Charlson comorbidity index; PADT, primary androgen deprivation therapy; RP, radical prostatectomy; STD, standardized difference for covariate imbalance check (covariates are balanced if STD is $<0.1$ ). 


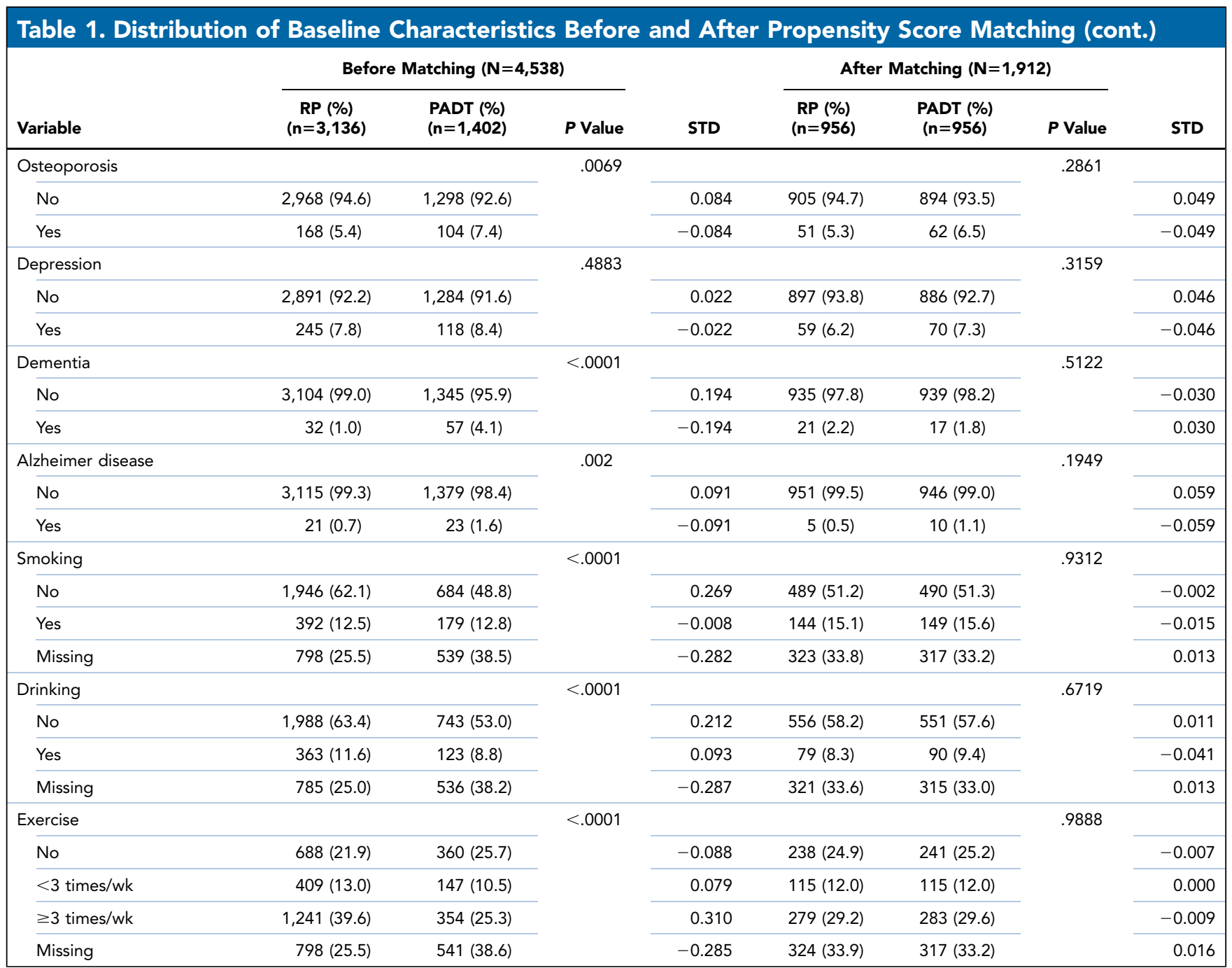

Abbreviations: BMI, body mass index; CCl, Charlson comorbidity index; PADT, primary androgen deprivation therapy; RP, radical prostatectomy; STD, standardized difference for covariate imbalance check (covariates are balanced if STD is $<0.1$ ).

Table 2 and supplemental eTable 3 present the Cox proportional hazards regression for overall survival of the RP and PADT groups in the pre- and post-PSM cohorts. Cox proportional hazards model revealed that, compared with men who underwent RP, those who received PADT experienced a greater than 4 -fold increased risk of mortality before matching after adjusting for all other covariates. In the cohort after PSM, the adjusted risk of mortality was significantly increased in the PADT group compared with the RP group. Table 3 presents the subgroup analysis stratified by stage and age in the postPSM cohort. Cox proportional hazards models for each subgroup revealed that men who received PADT had a significantly increased risk of death compared with those who underwent RP in every subgroup analyzed. In particular, a notably greater increase in risk was observed for patients with locally advanced prostate cancer, with a
5.42 -fold increased risk for those aged $<75$ years and a 3.16 -fold increased risk for those aged $\geq 75$ years.

According to $\mathrm{KM}$ estimates for subgroups of the post-PSM cohort, 5-year survival rates for patients aged $<75$ years with localized prostate cancer were $80.1 \%$ in the PADT group and $94.0 \%$ in the RP group, and were $75.0 \%$ and $84.2 \%$ for patients aged $\geq 75$ years in the PADT and RP cohorts, respectively. In the subgroup with locally advanced cancer, 5-year survival rates for patients aged $<75$ years were $75.2 \%$ in the PADT group and $94.3 \%$ in the RP group, and for those aged $\geq 75$ years were $65.5 \%$ in the PADT group and $87.5 \%$ in the RP group.

These results parallel the KM survival curves presented in Figures $3 \mathrm{~A}$ and 3B, which reveal better survival in the RP group than the PADT group for each stage subgroup (log-rank $P$ values for localized prostate cancer of $<.0001$ for patients aged $<75$ years and .0513 for 
A
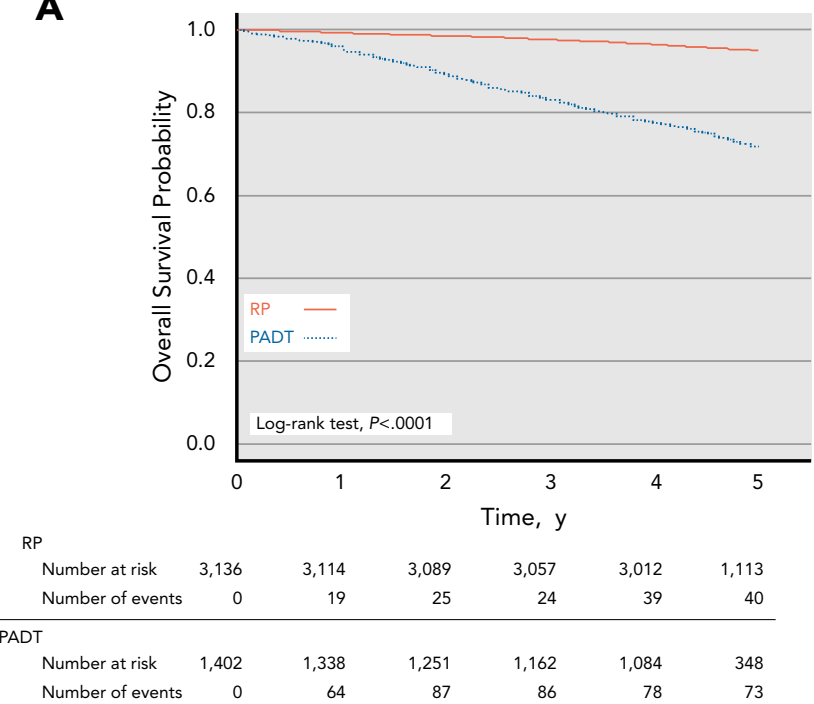

B

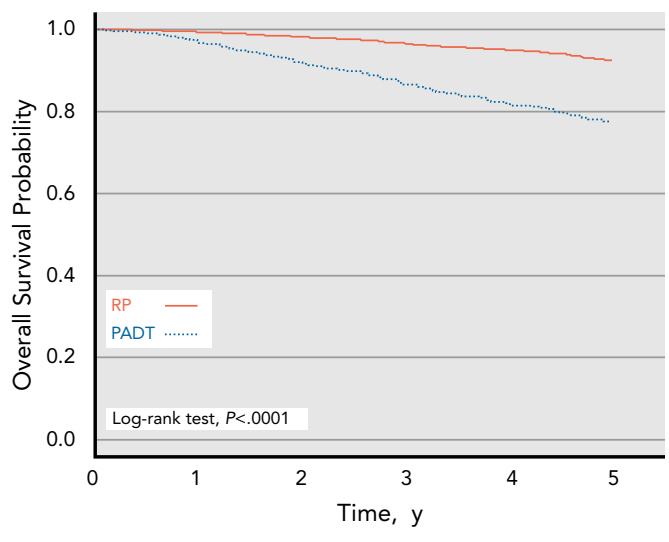

\begin{tabular}{lrrrrrr} 
Number at risk & 956 & 947 & 936 & 921 & 905 & 460 \\
Number of events & 0 & 8 & 11 & 14 & 15 & 22 \\
\hline PADT & & & & & & \\
Number at risk & 956 & 925 & 879 & 825 & 779 & 392 \\
$\quad$ Number of events & 0 & 31 & 46 & 51 & 46 & 38
\end{tabular}

Figure 2. Kaplan-Meier curves of overall survival in (A) pre-PSM and (B) post-PSM cohorts comparing PADT and RP groups. Abbreviations: PADT, primary androgen deprivation treatment; PSM, propensity score matching; RP, radical prostatectomy.

those aged $\geq 75$ years, and log-rank $P$ values for locally advanced prostate cancer $<.0001$ for patients aged $<75$ years and .0548 for those aged $\geq 75$ years).

\section{Discussion}

The main findings of this population-based study include that men who underwent RP had longer survival than those who received PADT. Treatment with RP was associated with a significantly lower risk of mortality compared with PADT after controlling for survival predictors. Second, compared with PADT, RP provided good clinical outcomes for patients with advanced prostate cancer in the older age group (aged $\geq 75$ years). Lastly, significant evidence favored RP over PDAT in determination of treatment direction in Asian populations. However, the lack of information regarding detailed tumor characteristics that may have affected survival outcomes should be considered.

Until recently, PADT for patients with nonmetastatic prostate cancer had not been proven to be effective in reducing the risk of prostate cancer mortality, ${ }^{3,13,14}$ and many studies reported that RP significantly reduced the risk of mortality and provided good long-term clinical outcomes in this patient population..$^{5,15-17}$ However, in actual practice, a considerable number of patients with clinically nonmetastatic prostate cancer are managed with PADT only. In a cohort analysis based on the SEERMedicare linked database, $>20 \%$ of cases of clinical T1 -2 prostate cancer were managed with PADT, and $>30 \%$ of patients with clinical T3 prostate cancer received PADT between 1998 and 2002. ${ }^{18}$ These guideline-discordant PADT patterns remained until recently, and one study reported that $12.4 \%$ of patients with localized disease received guideline-discordant PADT. ${ }^{19}$ This trend was also observed in our study, in which $>30 \%$ of patients with clinically nonmetastatic prostate cancer $(1,402$ of 4,538 patients) received PADT for primary treatment without $\mathrm{RP}$ or radiation treatment.

Although there are several reasons, including cost and complications, for choosing primary PADT instead of RP despite discordance with guidelines, age, general condition, and comorbidities could also act as obstacles to general anesthesia for surgery, leading physicians and patients to select PADT. Among these variables, older age has been considered an important factor discouraging

Table 2. Cox Proportional Hazards Regression for Overall Survival Before and After PSM

\begin{tabular}{|c|c|c|c|c|c|c|c|c|}
\hline \multirow[b]{2}{*}{ Variable } & \multirow[b]{2}{*}{$\mathbf{N}$ (Events) } & \multicolumn{3}{|c|}{ Before Matching ( $N=4,538)$} & \multirow[b]{2}{*}{$\mathbf{N}$ (Events) } & \multicolumn{3}{|c|}{ After Matching $(\mathrm{N}=1,912)$} \\
\hline & & HR & $95 \% \mathrm{Cl}$ & $P$ Value & & HR & $95 \% \mathrm{Cl}$ & $P$ Value \\
\hline RP & $3,136(147)$ & 1 & Ref & & $956(70)$ & 1 & Ref & \\
\hline PADT & $1,402(389)$ & 4.52 & $(3.66-5.57)$ & $<.0001$ & 956 (213) & 3.42 & (2.61-4.48) & $<.0001$ \\
\hline
\end{tabular}

Multivariable adjusted models controlled for the following variables: age (in years), Charlson comorbidity index $(0,1,2,3, \geq 4)$, body mass index, hypertension (yes or no), hyperlipidemia (yes or no), osteoporosis (yes or no), depression (yes or no), smoking (yes or no), drinking (yes or no), and exercise (yes or no).

Abbreviations: HR, hazard ratio; PADT, primary androgen deprivation therapy; PSM, propensity score matching; RP, radical prostatectomy. 


\section{Table 3. Cox Proportional Hazards Regression Analysis for Overall Survival in the PSM Cohort}

\begin{tabular}{|c|c|c|c|c|c|c|c|c|c|c|}
\hline Variable & \multicolumn{5}{|c|}{ Localized $(\mathrm{N}=1,392)$} & \multicolumn{5}{|c|}{ Locally Advanced ( $N=520)$} \\
\hline \multicolumn{11}{|l|}{ All ages } \\
\hline $\mathrm{RP}$ & $696(51)$ & 15.3 & 1.00 & Ref & & $260(19)$ & 15.4 & 1.00 & Ref & \\
\hline PADT & $696(145)$ & 47.1 & 3.42 & $(2.61-4.48)$ & $<.0001$ & $260(68)$ & 60.4 & 4.61 & $(2.71-7.83)$ & $<.0001$ \\
\hline \multicolumn{11}{|c|}{ Age $<75$ y } \\
\hline PADT & $575(115)$ & 44.8 & 3.79 & $(2.55-5.63)$ & $<.0001$ & $228(56)$ & 56.1 & 5.42 & $(2.90-10.15)$ & $<.0001$ \\
\hline \multicolumn{11}{|c|}{ Age $\geq 75 y$} \\
\hline $\mathrm{RP}$ & $121(19)$ & 33.7 & 1.00 & Ref & & $32(5)$ & 34.9 & 1.00 & Ref & \\
\hline PADT & $121(30)$ & 58.3 & 1.88 & $(1.04-3.39)$ & .0366 & $32(12)$ & 93.9 & 3.16 & $(0.91-10.95)$ & .0702 \\
\hline
\end{tabular}

Multivariable adjusted models controlled for the following variables: age (in y), Charlson comorbidity index $(0,1,2,3, \geq 4)$, body mass index, hypertension (yes or no), hyperlipidemia (yes or no), osteoporosis (yes or no), depression (yes or no), smoking (yes or no), drinking (yes or no), and exercise (yes or no).

Abbreviations: HR, hazard ratio; PADT, primary androgen deprivation therapy; PSM, propensity score-matched; RP, radical prostatectomy.

aPerson-year rate $=$ event/person year $\times 1,000$.

surgeons and patients from surgery. ${ }^{6,20,21}$ Baseline characteristics in our study cohort without PSM revealed a clear distinction in mean age between the PADT and RP groups.

In addition, in a study based on a Japanese population, Matsumoto et $\mathrm{al}^{22}$ conducted a survival analysis of PADT in patients with localized prostate cancer and compared their life expectancy with that of the normal population. Their results suggested that PADT might represent a therapeutic option for localized intermediateand high-risk prostate cancer in older men, because overall survival after PADT was not inferior to the expected survival of the normal population. Another study conducted in Japan demonstrated a survival benefit for PADT. ${ }^{7,8}$ Although some of these results served as the basis for the NCCN Asia Consensus Statement regarding the role of PADT, only one study ${ }^{7}$ has directly compared PADT and RP.

However, based on that one study, ${ }^{7}$ it is difficult to unreservedly accept the conclusion that PADT is noninferior to RP with respect to improving survival rate
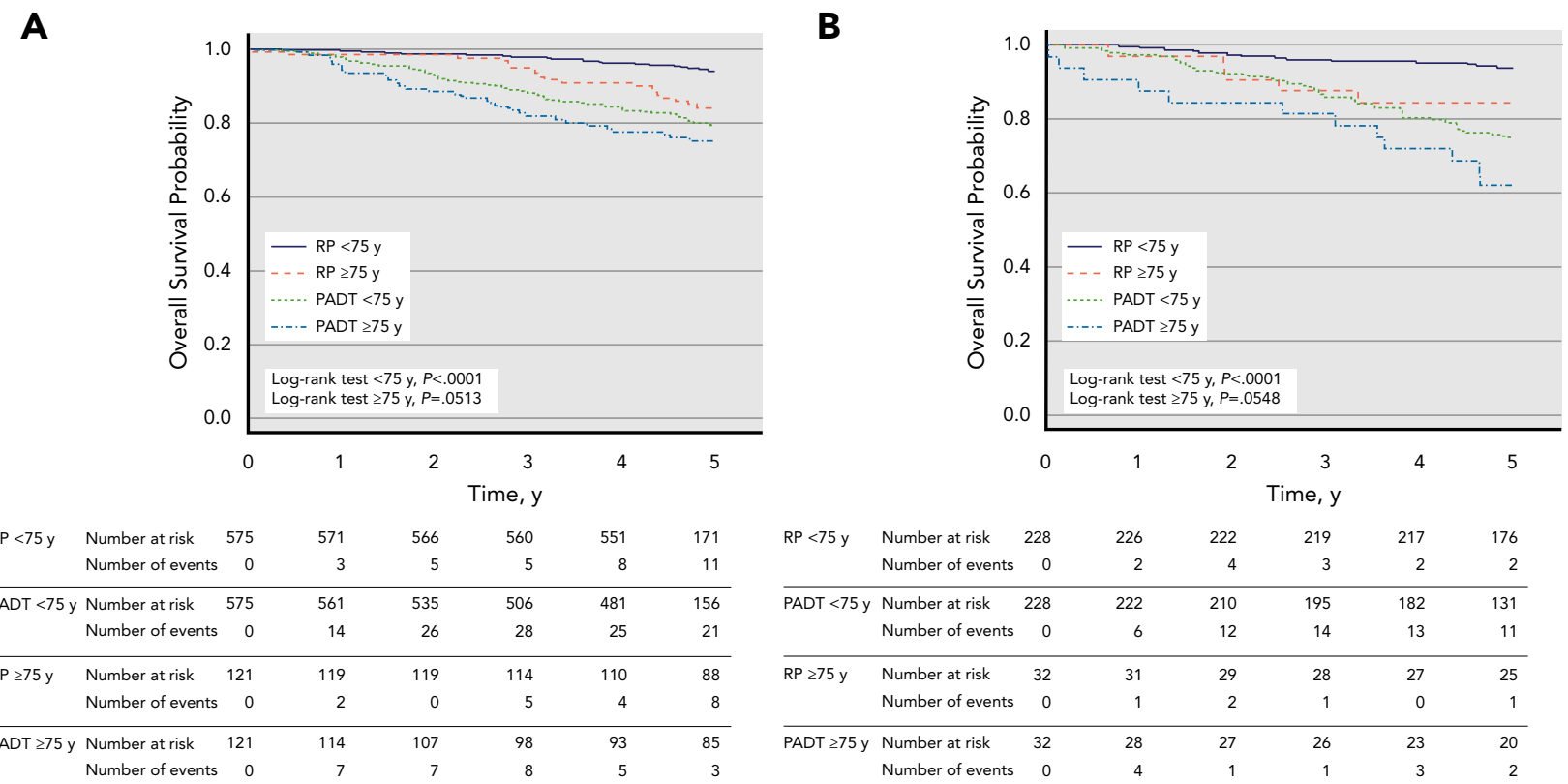

Figure 3. Kaplan-Meier curves of overall survival in propensity score-matched cohorts comparing PADT and RP groups for (A) localized disease and (B) locally advanced disease among patients aged $<75$ and $\geq 75$ years.

Abbreviations: PADT, primary androgen deprivation treatment; RP, radical prostatectomy. 
and life expectancy, because enrolled patients were not randomly allocated to the RP and PADT groups, and comorbidities that affect survival were also not controlled for. Death due to other causes was relatively higher in the PADT group (44\%; 67 of 151) than in the RP group (23.2\%; 41 of 176); therefore, the sample size that could be used to determine the effect of prostate cancer treatment was greatly reduced. This concern may be a drawback to using that cohort to determine the efficacy of PADT, even if the negative effects of PADT, including cardiovascular effects, are excluded. These factors can partially explain discrepancies between the findings of this prior study and our results. In fact, a Japanese study comparing RP and PADT showed that the overall survival rate was lower in the PADT group than in the RP group for patients aged $<70$ years (5-year survival: $84 \%$ vs $90 \%$, respectively, and 10 -year survival: $73 \%$ vs $85 \%$, respectively). Therefore, it is difficult to accept the prior investigation, which concluded that PADT was noninferior to $\mathrm{RP}$, as a representative study with respect to developing guidelines.

Thus, it is noteworthy that our study, based on the Korean population, revealed that treatment with RP provided a significant survival advantage among patients with prostate cancer compared with PADT, especially in patients aged $\geq 75$ years with locally advanced disease. Our finding that RP can provide better survival benefits than PADT in nonmetastatic prostate cancer even in an Asian population may strengthen the current guidelines.

The distinctive feature of our study is that, to the best of our knowledge, it is the first study directly comparing $\mathrm{RP}$ with PADT in an Asian population of patients with nonmetastatic prostate cancer stratified by age and stage. Few data are available comparing PADT with RP in men with nonmetastatic localized or locally advanced prostate cancer (cT1-T3,N0,M0). Our study, based on nationwide data, not only contributes to the literature about survival after RP versus PADT but also provides clinical information and implications favoring RP rather than PADT in elderly patients with prostate cancer.

Another distinctive feature of our study is that it was conducted in Asian patients with prostate cancer. Cardiovascular complications after ADT are a concerning factor that can affect survival. However, ADT seems to be better tolerated in Japanese populations than in Western cohorts ${ }^{23}$; therefore, it can be assumed that the negative effect of PADT on survival is relatively smaller in the Asian population, making it worthwhile to compare treatments. In addition, it should be noted that this is a comparative study between RP and PADT in an Asian population that contains older people with advanced disease.

The NHIS database system provides valuable nationwide data, but important limitations that could have affected our outcome analysis should be noted. This database does not include detailed biochemical information, such as Gleason grade and prostate-specific antigen, and therefore we could not evaluate the impact of those factors. Gleason grade and scoring are used to determine the aggressiveness of prostate cancer, and therefore this information has an impact on prognosis and treatment decisions. A lack of detailed information about tumor characteristics can introduce bias regarding both survival outcomes and treatment method decisions. Furthermore, in such a large population registry dataset, there may be concern about misclassification of data. However, patients with cancer enrolled in the NHIS database system are subject to a rigorous screening process given the large burden (50\%-95\%) of incurred medical costs. Therefore, the probability of misclassification is very low in this dataset compared with other registry datasets. Nonetheless, we may have missed capturing potentially important prognostic information, such as stage and other confounding factors that affect mortality.

Another limitation that must be carefully considered is an inability to distinguish whether mortality differences are due to prostate cancer-specific mortality or other-cause mortality. To address and minimize factors that could affect survival, we attempted to control for comorbidities using PSM involving diverse risk factors, including CCI, accompanying diseases, BMI, and personal behaviors associated with individual health (eg, drinking and smoking), and we used different types of variables in the statistical model. However, it is evident that these analytical methods cannot sufficiently address the aforementioned limitations. Moreover, when comparing the efficacy of treatment methods, caution must be exercised when drawing a conclusion based only on overall mortality. Therefore, there are certain drawbacks to reaching firm conclusions, and we must consider the possibility that these drawbacks caused the overestimation of survival differences before accepting the findings of this study. To make our findings more reliable, further analysis is needed that includes more detailed information regarding tumor characteristics.

\section{Conclusions}

Our study provides a nationwide comparative analysis of survival after RP versus PADT in Asian patients with nonmetastatic prostate cancer. We found that men who underwent RP had longer survival than those who received PADT in this cohort and in the subgroup of older patients with advanced prostate cancer. Because we could not completely control for confounding factors owing to a lack of tumor characteristics, such as Gleason grade, we are reluctant to conclude that we have 
sufficient evidence to demonstrate that RP provided a survival benefit compared with PADT. However, these findings provide valuable clinical implications that favor RP in decisions regarding prostate cancer treatment in Asian populations.

Submitted April 13, 2018; accepted for publication December 20, 2018

Author contributions: Study concept and design: Ha, J.B. Choi, E. Park, Ahn, J.Y. Lee. Sample recruitment: Shim, M. Kang, S. Kang, J. Park, Yang, I. Choi, Kwak, Jeong, Kim, Byun, Seo, H.M. Lee, S.J. Lee, S.H. Lee, Chung. Data analysis: Shim, M. Kang, E. Park, S. Kang, J. Park, Yang, I. Choi. Manuscript preparation: Ha, J.B. Choi, E. Park, J.Y. Lee. Critical revision and final approval: All authors.

Disclosures: The authors have not received any financial consideration from any person or organization to support the preparation, analysis, results, or discussion of this article.

Funding: Supported by the National Evidence-based Healthcare Collaborating Agency (NECA), which is funded by the Ministry of Health and Welfare (NC16-003 [J.Y.L.]), and the Bio \& Medical Technology Development Program of the Nationa Research Foundation (NRF), which is funded by the Korean government (MSIT) (NRF-2017M3A9B8069577 [J.Y.L.])

Correspondence: Ji Youl Lee, MD, PhD, Department of Urology, Seoul St. Mary's Hospital, College of Medicine, The Catholic University of Korea, 222 Banpo-daero, Seocho-gu, Seoul, 06591, Republic of Korea.

Email: uroljy@catholic.ac.kr

\section{References}

1. Mottet N, Bellmunt J, Briers E, et al. EAU-ESTRO-ESUR-SIOG guidelines on prostate cancer. Presented at the 2018 EAU Annual Congress; Copenhagen, Denmark; March 16-20, 2018. Available at: https:// uroweb.org/wp-content/uploads/EAU-ESUR-ESTRO-SIOG-Guidelineson-Prostate-Cancer-large-text-V2.pdf. Accessed January 1, 2019.

2. Mohler JL, Lee RJ, Antonarakis ES, et al. NCCN Clinical Practice Guidelines in Oncology: Prostate Cancer. Version 3.2018. To view the most recent version of these guidelines, visit NCCN.org. Accessed July 12, 2018.

3. Lu-Yao GL, Albertsen PC, Moore DF, et al. Survival following primary androgen deprivation therapy among men with localized prostate cancer. JAMA 2008;300:173-181.

4. Akaza $\mathrm{H}$. Trends in primary androgen depletion therapy for patients with localized and locally advanced prostate cancer: Japanese perspective. Cancer Sci 2006:97:243-247.

5. Stephenson AJ, Kattan MW, Eastham JA, et al. Prostate cancerspecific mortality after radical prostatectomy for patients treated in the prostate-specific antigen era. J Clin Oncol 2009;27:4300-4305.

6. Cooperberg MR, Grossfeld GD, Lubeck DP, et al. National practice patterns and time trends in androgen ablation for localized prostate cancer. J Natl Cancer Inst 2003;95:981-989.

7. Akaza $\mathrm{H}$, Homma $\mathrm{Y}$, Usami M, et al. Efficacy of primary hormone therapy for localized or locally advanced prostate cancer: results of a 10-year follow-up. BJU Int 2006;98:573-579.

8. Ueno S, Namiki M, Fukagai T, et al. Efficacy of primary hormonal therapy for patients with localized and locally advanced prostate cancer: a retrospective multicenter study. Int J Urol 2006;13:1494-1500.

9. Cooperberg MR, Hinotsu S, Namiki M, et al. Trans-Pacific variation in outcomes for men treated with primary androgen-deprivation therapy (ADT) for prostate cancer. BJU Int 2016;117:102-109.

10. Akaza $\mathrm{H}$. Future prospects for luteinizing hormone-releasing hormone analogues in prostate cancer treatment. Pharmacology 2010;85:110-120.

11. Akaza H, Umbas R, Cheng JCH, et al. NCCN Clinical Practice Guidelines in Oncology-Asia Consensus Statement: Prostate Cancer. Version 2.2013. To view the most recent version of these guidelines, visit NCCN.org Accessed July 12, 2018.

12. Ozono S, Hinotsu S, Namiki M, et al. NCCN Asian consensus statement can Asian patients with cancer accept treatment modalities from NCCN guidelines [in Japanese]? Gan To Kagaku Ryoho 2014;41:683-686.
13. Potosky AL, Haque R, Cassidy-Bushrow $A E$, et al. Effectiveness of primary androgen-deprivation therapy for clinically localized prostate cancer. J Clin Oncol 2014;32:1324-1330.

14. Kuo YF, Montie JE, Shahinian VB. Reducing bias in the assessment of treatment effectiveness: androgen deprivation therapy for prostate cancer. Med Care 2012;50:374-380.

15. Holmberg L, Bill-Axelson A, Steineck G, et al. Results from the Scandinavian Prostate Cancer Group Trial number 4: a randomized controlled trial of radical prostatectomy versus watchful waiting J Natl Cancer Inst Monogr 2012;2012:230-233.

16. Gandaglia G, Sun M, Trinh $Q D$, et al. Survival benefit of definitive therapy in patients with clinically advanced prostate cancer: estimations of the number needed to treat based on competing-risks analysis. BJU Int 2014;114:E62-E69.

17. Bill-Axelson A, Holmberg L, Garmo H, et al. Radical prostatectomy or watchful waiting in early prostate cancer. N Engl J Med 2014;370: 932-942.

18. Shahinian VB, Kuo YF, Freeman JL, et al. Determinants of androgen deprivation therapy use for prostate cancer: role of the urologist. J Nat Cancer Inst 2006;98:839-845.

19. Kuykendal AR, Hendrix LH, Salloum RG, et al. Guideline-discordant androgen deprivation therapy in localized prostate cancer: patterns of use in the medicare population and cost implications. Ann Oncol 2013;24: 1338-1343.

20. Cancer Registration Committee of the Japanese Urological Association Clinicopathological statistics on registered prostate cancer patients in Japan: 2000 report from the Japanese Urological Association. Int J Uro 2005;12:46-61.

21. Schymura MJ, Kahn AR, German RR, et al. Factors associated with initial treatment and survival for clinically localized prostate cancer: results from the CDC-NPCR Patterns of Care Study (PoC1). BMC Cancer 2010;10:152.

22. Matsumoto K, Hagiwara M, Tanaka N, et al. Survival following primary androgen deprivation therapy for localized intermediate- or high-risk prostate cancer: comparison with the life expectancy of the agematched normal population. Med Oncol 2014;31:979.

23. Kitagawa $\mathrm{Y}$, Ueno $\mathrm{S}$, Konaka $\mathrm{H}$, et al. Experience with androgen deprivation therapy for prostate cancer in Japan and future perspectives. Curr Cancer Drug Targets 2015;15:314-326. 
Supplemental online content for:

\section{Is Primary Androgen Deprivation Therapy a Suitable Option for Asian Patients With Prostate Cancer Compared With Radical Prostatectomy?}

U-Syn Ha, MD, PhD; Jin Bong Choi, MD; Jung Im Shim, MS; Minjoo Kang, MS; Eunjung Park, PhD; Shinhee Kang, PhD; Jooyeon Park, MS; Jangmi Yang, MS; Insun Choi, PhD; Jeonghoon Ahn, PhD; Cheol Kwak, MD, PhD; Chang Wook Jeong, MD, PhD; Choung Soo Kim, MD, PhD; Seok-Soo Byun, MD, PhD; Seong II Seo, MD, PhD; Hyun Moo Lee, MD, PhD; Seung-Ju Lee, MD, PhD; Seung Hwan Lee, MD, PhD; Byung $\mathrm{Ha}$ Chung, MD, PhD; and Ji Youl Lee, MD, PhD

J Natl Compr Canc Netw 2019;17(5):441-449

eTable 1: Distribution of Baseline Characteristics Before and After Propensity Score Matching for Localized Disease eTable 2: Distribution of Baseline Characteristics Before and After Propensity Score Matching for Locally Advanced Disease

eTable 3: Cox Proportional Hazards Regression for Overall Survival Before and After Propensity Score Matching 


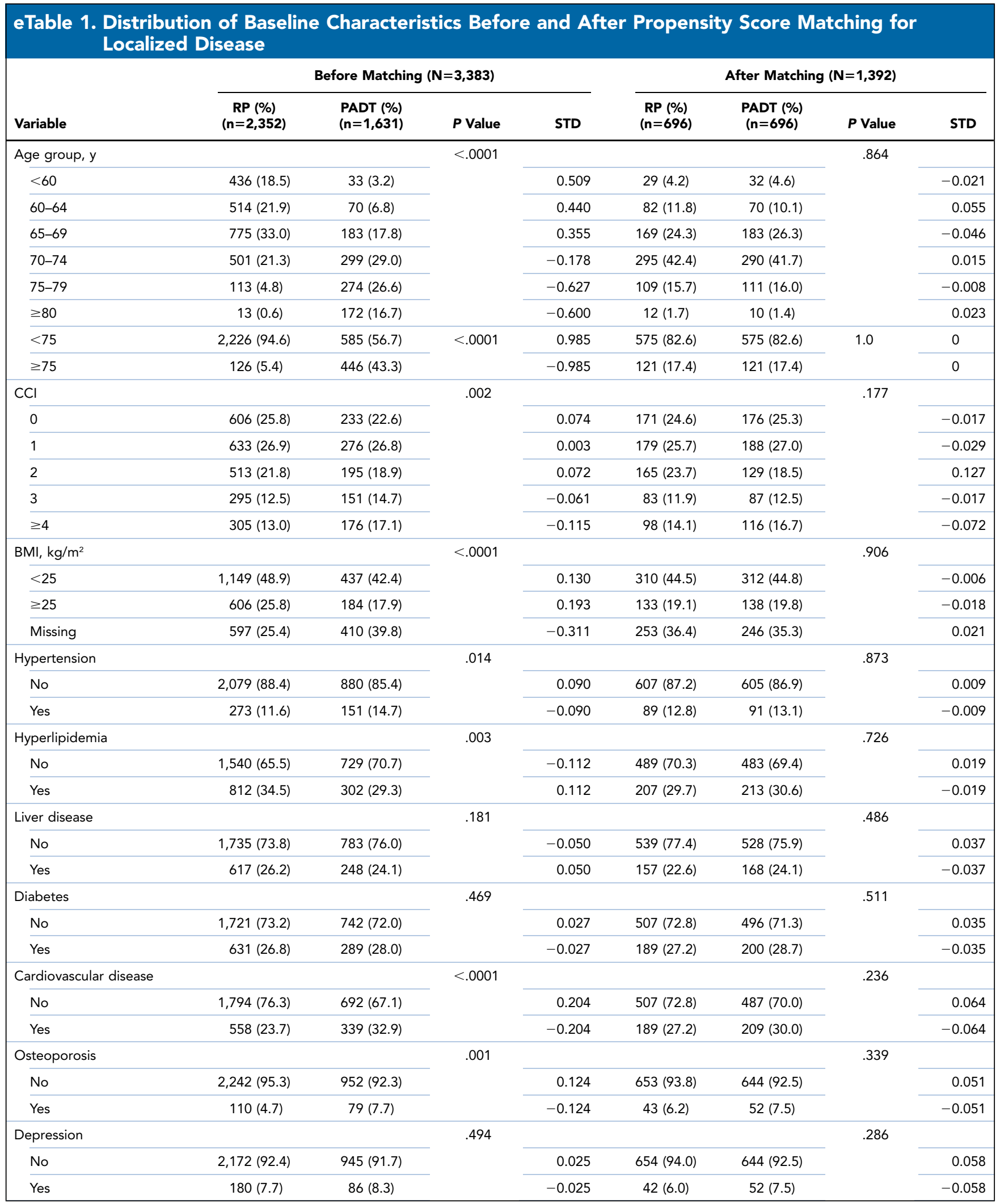




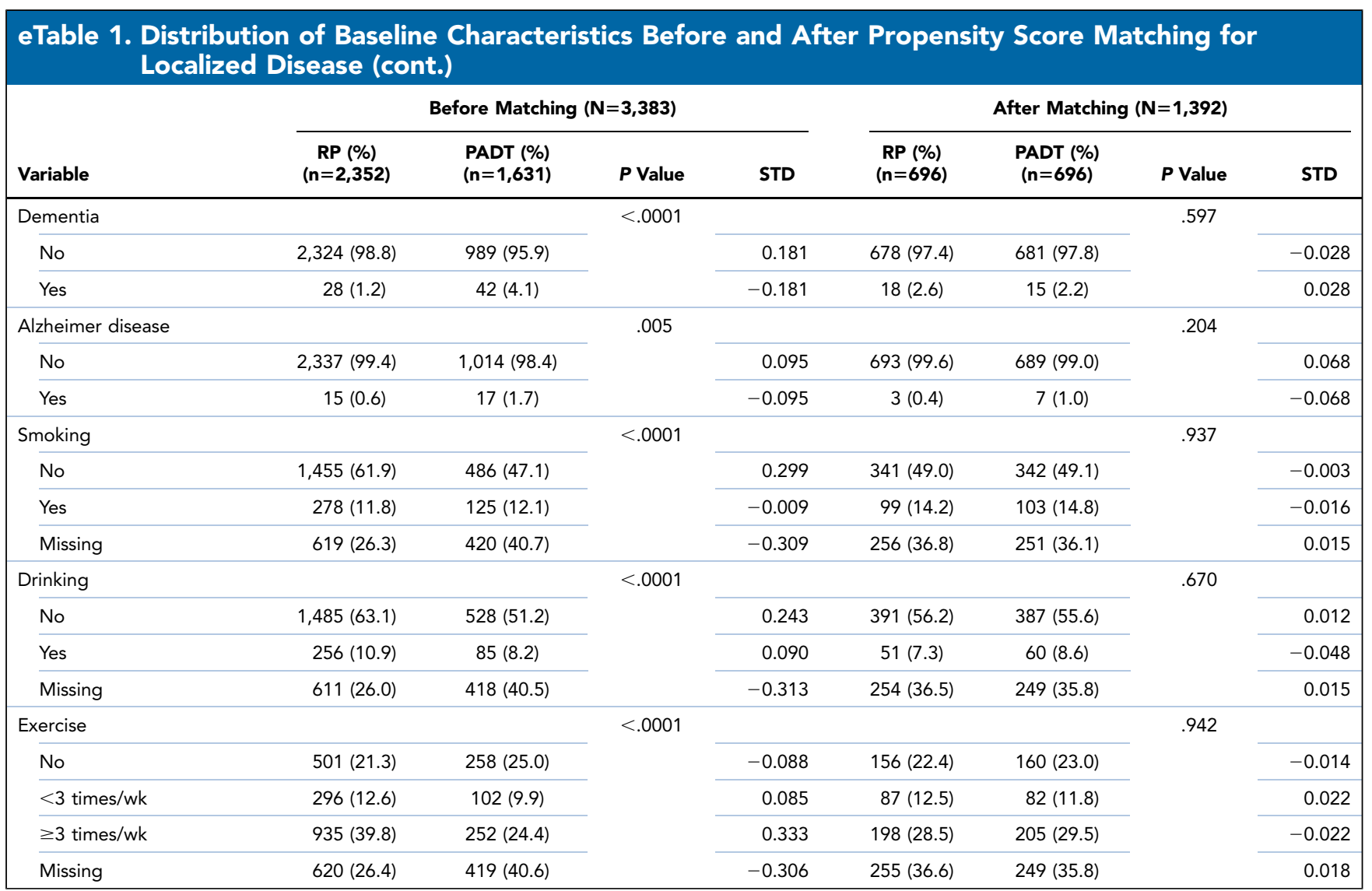

Abbreviations: BMI, body mass index; CCl, Charlson comorbidity index; PADT, primary androgen deprivation therapy; RP, radical prostatectomy; STD, standardized difference for covariate imbalance check (covariates are balanced if STD is $<0.1$ ). 
eTable 2. Distribution of Baseline Characteristics Before and After Propensity Score Matching for Locally Advanced Disease

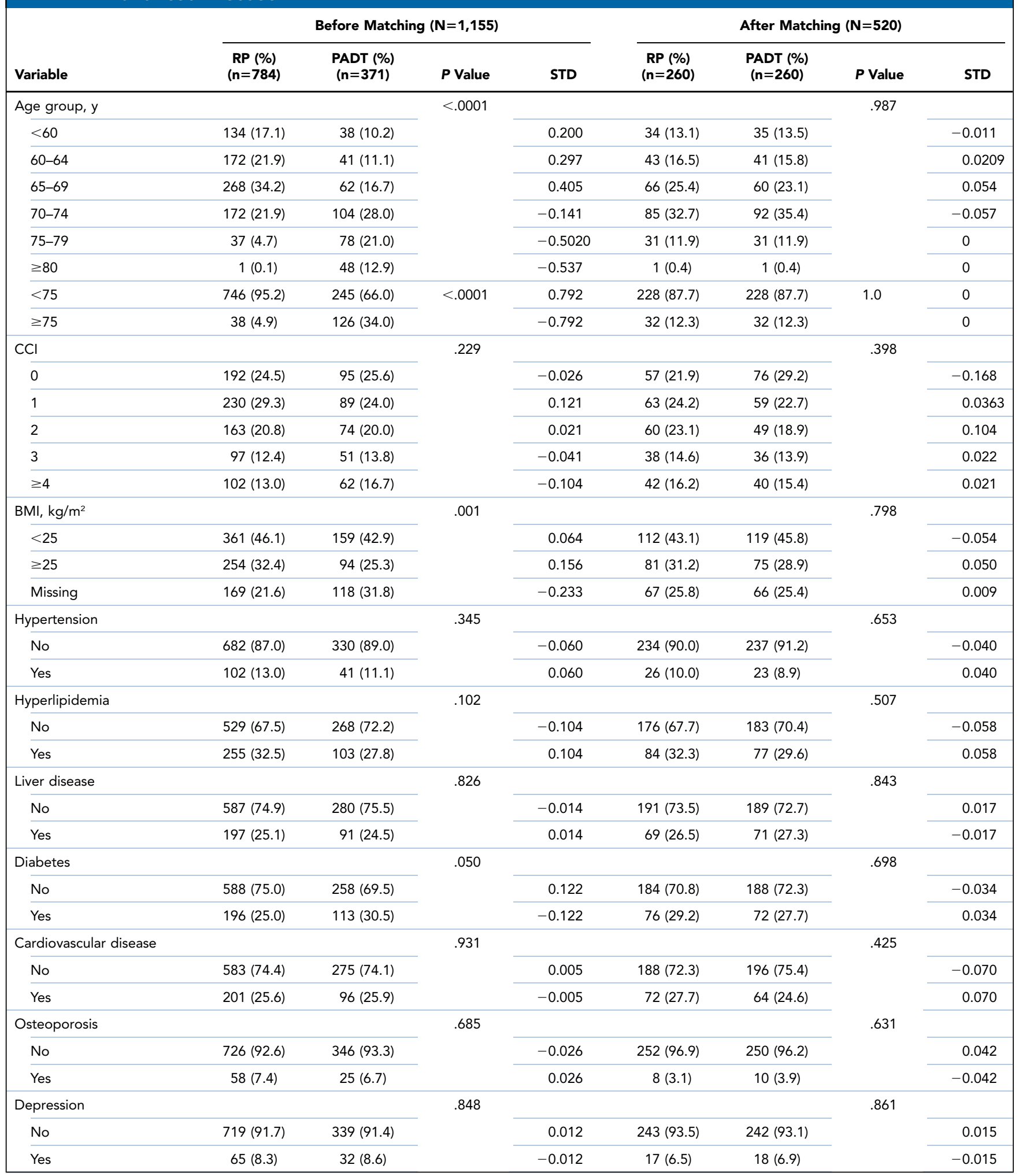

Abbrevintinued on next page) Abbreviations: BMI, body mass index; CCl, Charlson comorbidity index; PADT, primary androgen deprivation treatment; RP, radical prostatectomy; STD, standardized difference for covariate imbalance check (covariates are balanced if STD is $<0.1$ ). 


\begin{tabular}{|c|c|c|c|c|c|c|c|c|}
\hline \multirow[b]{2}{*}{ Variable } & \multicolumn{4}{|c|}{ Before Matching ( $N=1,155)$} & \multicolumn{4}{|c|}{ After Matching ( $N=520)$} \\
\hline & $\begin{array}{c}\mathrm{RP}(\%) \\
(n=784)\end{array}$ & $\begin{array}{c}\text { PADT (\%) } \\
(n=371)\end{array}$ & $P$ Value & STD & $\begin{array}{c}\operatorname{RP}(\%) \\
(n=260)\end{array}$ & $\begin{array}{c}\text { PADT (\%) } \\
(n=260)\end{array}$ & $P$ Value & STD \\
\hline \multicolumn{3}{|l|}{ Dementia } & $<.0001$ & & & & .653 & \\
\hline No & $780(99.5)$ & $356(96.0)$ & & 0.239 & $257(98.9)$ & $258(99.2)$ & & -0.039 \\
\hline Yes & $4(0.5)$ & $15(4.0)$ & & -0.239 & $3(1.2)$ & $2(0.8)$ & & 0.039 \\
\hline \multicolumn{3}{|l|}{ Alzheimer disease } & .182 & & & & .653 & \\
\hline No & $778(99.2)$ & $365(98.4)$ & & 0.079 & $258(99.2)$ & 257 (98.9) & & 0.039 \\
\hline Yes & $6(0.8)$ & $6(1.6)$ & & -0.079 & $2(0.8)$ & $3(1.2)$ & & -0.039 \\
\hline \multicolumn{3}{|l|}{ Smoking } & .003 & & & & .991 & \\
\hline No & $491(62.6)$ & $198(53.4)$ & & 0.188 & $148(56.9)$ & $148(56.9)$ & & 0 \\
\hline Yes & $114(14.5)$ & $54(14.6)$ & & -0.000 & $45(17.3)$ & $46(17.7)$ & & -0.010 \\
\hline Missing & $179(22.8)$ & $119(32.1)$ & & -0.208 & $67(25.8)$ & $66(25.4)$ & & 0.009 \\
\hline \multicolumn{3}{|l|}{ Drinking } & .001 & & & & .961 & \\
\hline No & $503(64.2)$ & $215(58.0)$ & & 0.128 & $165(63.5)$ & $164(63.1)$ & & 0.008 \\
\hline Yes & $107(13.7)$ & $38(10.2)$ & & 0.105 & $28(10.8)$ & $30(11.5)$ & & -0.024 \\
\hline Missing & $174(22.2)$ & $118(31.8)$ & & -0.218 & $67(25.8)$ & $66(25.4)$ & & 0.009 \\
\hline \multicolumn{3}{|l|}{ Exercise } & $<.0001$ & & & & .923 & \\
\hline No & $187(23.9)$ & $102(27.5)$ & & -0.083 & $82(31.5)$ & $81(31.2)$ & & 0.008 \\
\hline$<3$ times/wk & $113(14.4)$ & $45(12.1)$ & & 0.067 & $28(10.8)$ & $33(12.7)$ & & -0.060 \\
\hline$\geq 3$ times $/ w k$ & $306(39.0)$ & $102(27.5)$ & & 0.247 & 81 (31.2) & $78(30.0)$ & & 0.025 \\
\hline Missing & $178(22.7)$ & $122(32.9)$ & & -0.229 & $69(26.5)$ & $68(26.2)$ & & 0.009 \\
\hline
\end{tabular}

Abbreviations: $\mathrm{BMI}$, body mass index; CCl, Charlson comorbidity index; PADT, primary androgen deprivation treatment; RP, radical prostatectomy; STD, standardized difference for covariate imbalance check (covariates are balanced if STD is $<0.1$ ). 


\section{eTable 3. Cox Proportional Hazards Regression for Overall Survival Before and After Propensity Score Matching}

\begin{tabular}{|c|c|c|c|c|c|c|c|c|}
\hline Variable & $\mathbf{N}$ (Events) & \multicolumn{3}{|c|}{ Before Matching $(\mathrm{N}=\mathbf{4 , 5 3 8 )}$} & $\mathbf{N}$ (Events) & \multicolumn{3}{|c|}{ After Matching $(\mathrm{N}=1,912)$} \\
\hline \multicolumn{9}{|l|}{ Age, y } \\
\hline$<75$ & $3,802(302)$ & 1 & Ref & & $1,606(217)$ & 1 & Ref & \\
\hline$\geq 75$ & 736 (234) & 1.91 & $(1.57-2.31)$ & $<.0001$ & $306(66)$ & 1.64 & $(1.24-2.18)$ & .0006 \\
\hline \multicolumn{9}{|l|}{ Cancer stage } \\
\hline Locally advanced & $1,155(153)$ & 1.25 & $(1.03-1.51)$ & .0213 & $520(87)$ & 1.41 & $(1.09-1.82)$ & .0099 \\
\hline \multicolumn{9}{|l|}{$\mathrm{CCl}$} \\
\hline 0 & $1,126(90)$ & 1 & Ref & & $480(57)$ & 1 & Ref & \\
\hline 1 & $1,128(123)$ & 1.30 & $(0.99-1.71)$ & .0588 & $489(64)$ & 1.24 & $(0.87-1.78)$ & .2367 \\
\hline 2 & 945 (108) & 1.68 & $(1.26-2.24)$ & .0004 & $403(54)$ & 1.49 & $(1.02-2.19)$ & .0397 \\
\hline No & 3,971 (459) & 1 & Ref & & $1,683(249)$ & 1 & Ref & \\
\hline Yes & $567(77)$ & 1.01 & $(0.79-1.29)$ & .9663 & $229(34)$ & 1.11 & $(0.76-1.59)$ & .6059 \\
\hline \multicolumn{9}{|l|}{ Hyperlipidemia } \\
\hline No & 3,066 (388) & 1 & Ref & & $1,331(206)$ & 1 & Ref & \\
\hline Yes & $1,472(148)$ & 0.68 & $(0.55-0.83)$ & .0002 & $581(77)$ & 0.64 & $(0.48-0.86)$ & .0029 \\
\hline \multicolumn{9}{|l|}{ Osteoporosis } \\
\hline No & $4,266(486)$ & 1 & Ref & & $1,799(258)$ & 1 & Ref & \\
\hline Yes & $272(50)$ & 1.41 & $(1.05-1.89)$ & .0234 & $113(25)$ & 1.60 & $(1.05-2.42)$ & .0273 \\
\hline \multicolumn{9}{|l|}{ Depression } \\
\hline$<3$ times/wk & $2,731(244)$ & 1 & Ref & & $1,107(130)$ & 1 & Ref & \\
\hline$\geq 3$ times/wk & $486(45)$ & 0.97 & $(0.70-1.34)$ & .8508 & $169(25)$ & 1.10 & $(0.71-1.70)$ & .6694 \\
\hline \multicolumn{9}{|l|}{ Exercise } \\
\hline No & $1,048(129)$ & 1 & Ref & & $479(67)$ & 1 & Ref & \\
\hline$<3$ times $/$ wk & $556(44)$ & 0.78 & $(0.55-1.11)$ & .1566 & $230(23)$ & 0.74 & $(0.46-1.19)$ & .2108 \\
\hline$\geq 3$ times/wk & 1,595 (109) & 0.72 & $(0.56-0.94)$ & .0142 & $562(61)$ & 0.77 & $(0.55-1.09)$ & .1469 \\
\hline \multicolumn{9}{|l|}{ BMI, $\mathrm{kg} / \mathrm{m}^{2}$} \\
\hline$<25$ & $2,106(203)$ & 1 & Ref & & $853(113)$ & 1 & Ref & \\
\hline$\geq 25$ & $1,138(89)$ & 0.91 & $(0.70-1.17)$ & .4436 & $427(43)$ & 0.76 & $(0.53-1.08)$ & .1269 \\
\hline
\end{tabular}

Multivariable adjusted models controlled for the following variables: age (in y), $\mathrm{CCl}(0,1,2,3, \geq 4)$, BMI, hypertension (yes or no), hyperlipidemia (yes or no), osteoporosis (yes or no), depression (yes or no), smoking (yes or no), drinking (yes or no), and exercise (yes or no).

Abbreviations: BMI, body mass index; CCl, Charlson comorbidity index; HR, hazard ratio; PADT, primary androgen deprivation therapy; RP, radical prostatectomy. 